\title{
Caracterización clínico patológica y hallazgos microbiológicos de la neutropenia febril en pacientes oncohematológicos en una clínica privada en la ciudad de Montería - Colombia
}

Clinicopathological characterization and microbiological findings of febrile neutropenia in oncohematological patients

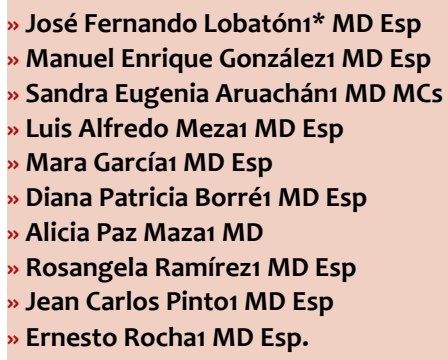

${ }^{1}$ Instituto Médico de Alta Tecnología, Montería - Colombia.

Recibido el 13 de agosto de 2020; Aceptado el 09 de septiembre de 2020

\section{Resumen}

Antecedentes: La neutropenia febril es una complicación frecuente en pacientes oncológicos que reciben quimioterapia. Se estima que el $50 \%$ de los pacientes con neoplasias sólidas y más del $80 \%$ con neoplasias hematológicas desarrollan neutropenia y fiebre en algún momento de su evolución.

Objetivo: Identificar las características clínico-patológicas, establecer los focos infecciosos y determinar el perfil microbiológico en los pacientes oncológicos que presentaron neutropenia febril, como complicación posquimioterapia y/o de terapias blanco en el Instituto Médico de Alta Tecnología (IMAT) de la ciudad de Montería - Colombia, entre junio de 2014 y junio de 2016.

Materiales y métodos: Estudio de tipo descriptivo en pacientes mayores de 18 años con diagnóstico de neoplasia hematológica o tumor sólido maligno, que presentaron neutropenia febril entre junio de 2014 y junio de 2016, que cumplían con los criterios de inclusión. El análisis de la información se realizó con el software $R$ versión 3.5.1.

Resultados: Se documentaron 76 casos de neutropenia febril. La edad promedio fue de 43 años y el $55 \%$ de los pacientes era de sexo masculino. El $84.2 \%$ de los casos se presentó en pacientes con neoplasias hematológicas, siendo las leucemias agudas las de mayor prevalencia. En el $68 \%$ se determinó el foco infeccioso; las infecciones del tracto respiratorio fueron el foco infeccioso clínico pre-

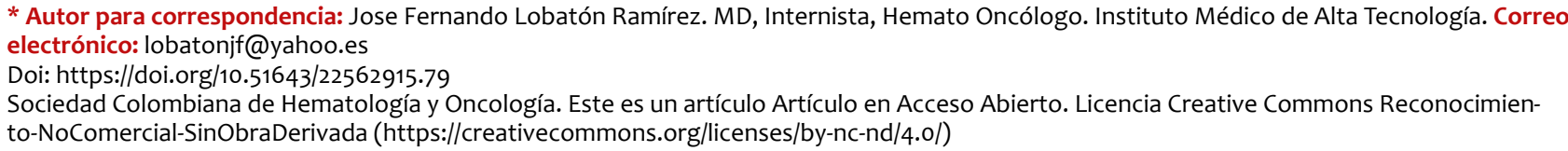


dominante (21\%). Los bacilos Gram negativos fueron los gérmenes más frecuentemente aislados.

Conclusión: Las infecciones del tracto respiratorio fueron el foco infeccioso predominante en los pacientes con neutropenia febril. Los bacilos Gram negativos fueron los principales microorganismos responsables. La tasa de mortalidad relacionada con neutropenia febril fue del $14 \%$, resultados similares a lo descrito en la población colombiana y a nivel mundial.

Palabras claves: Neutropenia; microbiología; quimioterapia; mortalidad.

\section{Abstract}

Background: Febrile neutropenia is a frequent complication in cancer patients receiving chemotherapy. It is estimated that $50 \%$ of patients with solid malignancies and more than $80 \%$ with hematologic malignancies develop neutropenia and fever at some point in their evolution.

Objective: To identify the clinical-pathological characteristics, establish the infectious foci, determine the microbiological profile in oncological patients who present with febrile neutropenia as a post-chemotherapy complication and / or target therapies at the Instituto Medico de Alta Tecnología (IMAT) in the city of Montería - Colombia between June 2014 to June 2016.

Material and methods: This is a descriptive study; The study population corresponds to patients over 18 years of age with a diagnosis of hematological malignancy or solid malignant tumor presenting with febrile neutropenia between June 2014 and June 2016, who meet the inclusion criteria. Statistical analysis: the analysis of the information was carried out in software $R$ version 3.5.1).

Results: 76 cases of febrile neutropenia were documented. The average age was 43 years and $55 \%$ of the patients were male. $84.2 \%$ of cases occurred in patients with hematologic malignancies, acute leukemias being the most prevalent. Infectious focus was determined in $68 \%$, respiratory tract infections was the predominant clinical infectious focus (21\%). Gram negative bacilli were the most frequently isolated germs.

Conclusion: In this series, respiratory tract infections were the predominant infectious focus in patients with febrile neutropenia. Gram negative bacilli, the main responsible microorganisms. The mortality rate related to febrile neutropenia was 14\%, results similar to those described in the Colombian population and worldwide.

Keywords: Neutropenia; microbiology; chemotherapy; mortality.

\section{Introducción}

La neutropenia febril es una complicación frecuente en pacientes oncológicos que reciben quimioterapia. Se estima que el $50 \%$ de los pacientes con neoplasias sólidas y más del $80 \%$ con neoplasias hematológicas desarrollan neutropenia y fiebre en algún momento de su evolución ${ }^{1}$. La neutropenia febril se define como la temperatura corporal mayor de $38{ }^{\circ} \mathrm{C}$ sostenida por más de una hora o mayor que $38.3^{\circ} \mathrm{C}$ en una sola toma, asociada a un recuento de neutrófilos absolutos $<5000<1000$ con tendencia a disminuir en las siguientes 48 horas por debajo de $500^{1,2}$.

Con el aumento en la detección temprana de neoplasias por los programas de prevención y promoción de la salud y el inicio de protocolos de quimioterapia, ha aumentado la prevalencia de la neutropenia febril, con una mortalidad va- 
riable entre el 5 y el 40 \%, según la Asociación Multinacional de Cuidado de Soporte en Cáncer (MASCC) ${ }^{3}$.

La neutropenia febril es considerada como una urgencia infectológica y oncológica, en la que un tratamiento inicial puede cambiar el pronóstico del paciente. El manejo apropiado implica contar con definiciones precisas y consensuadas: un protocolo de manejo propio de cada institución, con procesos definidos y eficientes que aseguren que el paciente va a ser reconocido precozmente, estudiado en forma apropiada; y un tratamiento inicial que va a ser iniciado rápidamente, idealmente entre los 3060 minutos de identificada la situación. La selección de la terapia empírica para pacientes con neutropenia febril suele apoyarse en las guías internacionales y en los patrones de resistencia a antibióticos locales ${ }^{4}$.

El perfil epidemiológico de las infecciones asociadas a neutropenia febril ha variado a través del tiempo. Al principio del desarrollo de la quimioterapia citotóxica, entre los años 1960 y 1970, predominaron los patógenos Gram negativos (60-70\%).

Luego, durante las décadas de 1980 y 1990, los cocos Gram positivos se hicieron más comunes (55-70 \%) debido al aumento en el uso de catéter venoso central que permitían la colonización y entrada de la flora cutánea. A partir de la mitad de la primera década del siglo XXI, nuevamente existe un predominio de bacilos Gram negativos. En un estudio realizado en el periodo 2004-2005 se observó una prevalencia del 61.9\% y del $38.1 \%$ para bacilos Gram negativas y cocos Gram positivos respectivamente ${ }^{5}$.

Algunas infecciones asociadas a neutropenia febril son causadas por hongos. Sin embargo, las infecciones micóticas son raramente identificadas como causa de fiebre en la primera semana; pueden ser la etiología en pacientes con neutropenia prolongada y profunda en más del $20 \%$. Se incluyen especies de Candida, ApergiIlus y Fusarium ${ }^{5}$.

La herramienta más útil para predecir complicaciones en neutropenia febril es el puntaje de MASCC, derivado de estudios multicéntricos, que se basan en antecedentes del paciente y hallazgos de la historia clínica, con un buen rendi- miento para predecir complicaciones. Los resultados superiores a 21 indican un bajo riesgo en los pacientes, con valores predictivos positivos del $91 \%$ y negativos del $36 \%$. Con este umbral, la sensibilidad es del $71 \%$ y la especificidad del $6 \%$. La mortalidad de los pacientes con neutropenia febril de acuerdo a este índice pronóstico es del $3 \%$ si la puntuación es mayor a 21, y del $36 \%$ si la puntuación es menor de $15^{1,6}$.

Los pacientes con neutropenia febril no presentan los síntomas usuales de infección localizada o sistémica que refieren los pacientes inmunocompetentes. La fiebre es a veces el único indicador del desarrollo de una infección y hasta el 50 \% de los pacientes con neutropenia febril se recuperan satisfactoriamente, sin que se encuentre un foco infeccioso aparente, ya sea por características clínicas, radiológicas o microbiológicas, con la terapia antimicrobiana empírica ${ }^{1,7}$.

Los pacientes con neutropenia febril considerados de alto riesgo según el puntaje de MASSC o con características de alto riesgo, deben ser hospitalizados para inicio de antibioticoterapia empírica. La monoterapia con un betalactámico con acción frente a Pseudomonas (Cefepime), un carbapenémico (Meropenem, Imipenen/cilastatina) o piperacilina tazobactan, son la primera línea recomendada de acuerdo a las directrices nacionales e internacionales. La adición de un antibiótico glucopéptido (Vancomicina) se indica en casos de mucositis severa, inestabilidad hemodinámica, neumonía documentada radiológicamente, infección relacionada con el catéter, infección de piel y tejidos blandos, colonización por MRSA, enterococos resistentes a Vancomicina (VRE) o Streptococcus pneumoniae resistente a penicilina.

La terapia empírica puede ser modificada a las 72 horas con los resultados de cultivos, indicando la utilización de Vancomicina, linezolid o daptomicina, en caso de cultivo positivo para MRSA o Linezolid o daptomicina para VRE y carbapenémicos para bacterias Gram negativas productoras de betalactamasa de espectro extendido (ESBL). Finalmente, ante cultivo positivo para organismos productores de carbapenemasas, incluyendo Klebsiella pneumoniae (KPC), considerar Polimixina-Colistina, Tigeci- 
clina o inhibidores de betalactamasas de última generación como ceftazidima/avibactam, aztreonam avibactam, meropenem varboctam y aztreonam relebactam ${ }^{2,3,8}$.

Si no hay respuesta a la modificación de la terapia, se debe examinar y repetir estudios de imágenes: tomografía axial computarizada (TAC) o resonancia nuclear magnética (RNM), para determinar nueva infección o progresión de la infección. Además de revisar la cobertura antimicrobiana, dosis y espectro, se debe considerar la adición de la terapia antifúngica empírica en aquellos pacientes con neutropenia prolongada y persistencia de fiebre luego de 96 horas de antiobioticoterapia de amplio espectro.

La terapia profiláctica con factores estimulantes de colonias de granulocitos, debe ser usada si el paciente tiene un riesgo anticipado de neutropenia $>20 \%$. La Sociedad Americana de Oncología (ASCO), la sociedad española de oncología médica (SEOM) y el National Comprehensive Cancer Network (NCCN) recomiendan el uso rutinario de factores estimulantes de colonias de granulocitos (G-CSF) en pacientes de alto riesgo. Definen el alto riesgo cuando la probabilidad de neutropenia es $\geq 20 \%$.

El NCCN define bajo riesgo cuando la probabilidad de neutropenia febril es $\leq 10 \%$. En estas situaciones, no se recomienda el empleo de factores estimulantes de colonias. En regímenes con riesgo intermedio, entendiendo por tal un riesgo de complicaciones neutropénicas entre un $10 \%$ y $20 \%$, se considera su uso como decisión individualizada al evaluar los factores de riesgo del paciente que puedan, a su vez, aumentar el riesgo de neutropenia febril ${ }^{9}$.

El propósito de este estudio fue establecer el perfil epidemiológico y microbiológico de la neutropenia febril en pacientes con neoplasias hematológicas y tumores sólidos malignos, que fueron atendidos en el Instituto Médico de Alta Tecnología Oncomédica S.A. en la ciudad de Montería - Colombia entre junio de 2014 y junio de 2016, el cual es centro de referencia para estas patologías en los departamentos de Córdoba y Sucre.

\section{Materiales y métodos}

Estudio descriptivo transversal retrospectivo. Se incluyeron pacientes $\geq 18$ años de edad con diagnóstico de enfermedad neoplásica maligna sólida o hematológica que consultaron en el IMAT en la ciudad de Montería - Colombia entre junio de 2014 y junio de 2016, con diagnóstico de neutropenia febril de acuerdo a las definiciones de la guía de la Sociedad Americana de Enfermedades Infecciosas y en tratamiento sistémico con quimioterapia o terapias blanco en las últimas tres semanas, previo a presentación de neutropenia febril. Se excluyeron sujetos con antecedentes de enfermedad del tejido conectivo, neutropenia asociada a infección por VIH, neutropenias sin patología hemato oncológicas, neutropenia inducida por fármacos, antecedente de aplasia medular y diagnóstico previo de mieloptisis.

La fuente de información fue la historia clínica sistematizada del IMAT entre junio de 2014 y junio de 2016, consultando reportes de laboratorios y resultados de estudios imagenológicos. Se revisaron las historias clínicas de los pacientes que cumplían con los criterios de inclusión, sin presencia de criterios de exclusión. Posteriormente, a través del programa de laboratorio clínico sistematizado se verificaron los resultados de los paraclínicos y específicamente de los hemocultivos.

Para el análisis estadístico se elaboró una matriz en Excel donde se consignaron las variables de estudio y una vez finalizada la recolección de la información, se exportó al programa estadístico Epi-Info versión 7.0. Se hizo el cálculo de medidas estadísticas de tendencia central y dispersión para variables cuantitativas y se determinaron pruebas de significancia estadística para variables categóricas como Chi-cuadrado y $T$ de Student para variables cuantitativas.

El estudio se realizó respetando los aspectos éticos de la normatividad nacional e internacional. Fue sometido y aprobado por el comité de ética institucional. Dado que la información se obtuvo de forma retrospectiva, no fue necesaria la implementación del consentimiento informado. 


\section{Resultados}

Se realizó el análisis de 91 ingresos de pacientes con neutropenia febril a la institución, de los cuales 76 casos cumplieron los criterios de inclusión. La Tabla 1 muestra las características de los pacientes.

El $45 \%$ de los casos se presentó en pacientes de sexo femenino y el $55 \%$ en pacientes de sexo masculino. Con respecto a la zona de procedencia, la mayoría de los casos eran procedentes del área urbana (63.2\%), mientras que el $36.8 \%$ de los pacientes eran residentes de zona rural. El tipo de régimen de afiliación de los pacientes correspondía en un $46.1 \%$ al régimen contributivo, en un $51.3 \%$ al régimen subsidiado y en un $2.6 \%$ a régimen especial. El promedio global de edad fue de 43 años, con una desviación estándar de 18 años.

Tabla 1. Características socio demográficas de los sujetos de estudio.

\begin{tabular}{|l|l|}
\hline Variables & $\begin{array}{l}\text { Casos neutropenia } \\
\text { febril }\end{array}$ \\
\hline Total casos & 76 \\
\hline Edad promedio & 43 años $( \pm 18)$ \\
\hline Sexo & $34(45 \%)$ \\
Femenino & $42(55 \%)$ \\
Masculino & $28(36.8 \%)$ \\
\hline Area procedencia & $48(63.2 \%)$ \\
Rural & $35(46.1 \%)$ \\
Urbana & $39(51.3 \%)$ \\
\hline Régimen salud & $2(2.6 \%)$ \\
Contributivo & Subsidiado \\
Especial &
\end{tabular}

Con relación a las características clínicas al diagnóstico de neutropenia febril, el $28 \%$ de los pacientes presentaba taquicardia y el $18 \%$ presentaba hipotensión. Se asoció mucositis en el $21 \%$ de los pacientes al diagnóstico. La temperatura promedio al diagnóstico fue de $38^{\circ} \mathrm{C}$.

Al diagnóstico de neutropenia febril, los hallazgos más relevantes del cuadro hemático fueron leucopenia, neutropenia moderada, anemia moderada a severa y trombocitopenia moderada. El conteo promedio de neutrófilos fue de $260.8 \times 10^{\wedge} 3 / \mu$ l, la severidad de la neutropenia al diagnóstico fue leve en el 16 \% de los casos, mo- derada en el $45 \%$ de los eventos y severa en el $39 \%$, siendo los valores promedio de neutrófilos para los casos leves de $742 \times 10^{\wedge} 3 / \mu$ l, en los casos moderados de $258.7 \times 10^{\wedge} 3 / \mu$ l y en los casos de neutropenia severa de $63.9 \times 10^{\wedge} 3 / \mu$, respectivamente.

Se evidenció anemia moderada a severa con una hemoglobina promedio de $8 \mathrm{~g} / \mathrm{dl}$ y una desviación estándar de $2.1 \mathrm{~g} / \mathrm{dl}$. La trombocitopenia fue un hallazgo de severidad moderada con un promedio de plaquetas de $80.965,3 \times 10^{\wedge} 3 / \mu \mathrm{l}$ con una desviación estándar de $85.168 \times 10^{\wedge} 3 / \mu$ l.

En el 68 \% de los de los casos se determinó el foco infeccioso de la neutropenia febril, y no se documentó en el $32 \%$ de los eventos. Los focos infecciosos más frecuentemente asociados a neutropenia febril fueron tracto respiratorio con $21 \%$ de los casos, de los cuales el $7 \%$ requirió manejo en $\mathrm{UCl}$; tracto digestivo con un $12 \%$ de incidencia y $1 \%$ de ingreso a UCl. Tejidos blandos y piel se presentó en el $12 \%$ de los casos, de los cuales el 3 \% ingresó a $\mathrm{UCl}$, seguido de tracto genitourinario con $9 \%$ de los casos, de los cuales ninguno requirió ingreso a $\mathrm{UCI}$. El $8 \%$ de los casos presentó un foco mixto y el 4 \% requirió ingreso a $\mathrm{UCl}$, el 6 \% de los casos presentó bacteremia sin foco infeccioso, de los cuales el 1 \% requirió ingreso a $\mathrm{UCl}$ (Figura 1).

Los casos de neutropenia febril se presentaron con mayor frecuencia en pacientes con neoplasias hematológicas (84.2\%), siendo las leucemias agudas las de mayor prevalencia, 29 $\%$ y $26 \%$ de los casos con leucemia linfoide aguda y leucemia mieloide aguda respectivamente; seguido por los pacientes con linfomas no

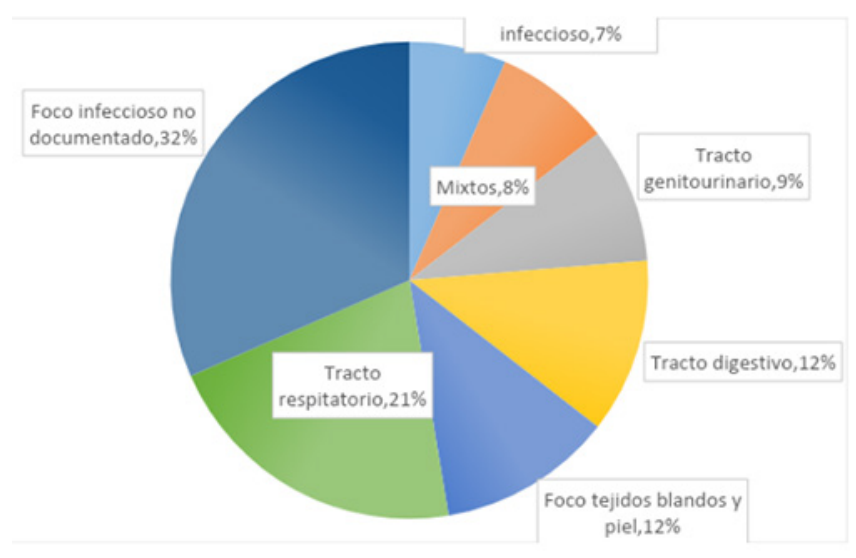


Hodgkin que representaron el $21 \%$ de los afectados por neutropenia febril. El $15.8 \%$ de los casos de neutropenia febril se desarrollaron en pacientes con neoplasias solidas malignas, siendo las más frecuentes el cáncer de mama y el cáncer gástrico con el 5 \% y el 3 \% de los casos, respectivamente (Tabla 2 ).

Tabla 2. Enfermedades neoplásicas presentes en los pacientes que desarrollan neutropenia febril.

\begin{tabular}{|c|c|c|c|}
\hline & Diagnóstico & Global & \\
\hline \multirow{7}{*}{$\begin{array}{c}\text { Neopl } \\
\text { asias } \\
\text { hemat } \\
\text { ológic } \\
\text { as }\end{array}$} & Leucemia Linfoide aguda & $29 \%$ & \multirow{7}{*}{$64(84,2 \%)$} \\
\hline & Leucemia Mieloide aguda & $26 \%$ & \\
\hline & Linfoma No Hodgkin & $21 \%$ & \\
\hline & Leucemia linfoide crónica & $3 \%$ & \\
\hline & Linfoma Hodgkin & $1 \%$ & \\
\hline & Mieloma múltiple & $1 \%$ & \\
\hline & Síndrome mielodisplásico & $1 \%$ & \\
\hline & Tricoleucemia & $1 \%$ & \\
\hline \multirow{8}{*}{$\begin{array}{c}\text { Neopl } \\
\text { asias } \\
\text { sólidas } \\
\text { malign } \\
\text { as }\end{array}$} & Cáncer mama- Colon & $5 \%$ & \multirow{8}{*}{$12(15.8 \%)$} \\
\hline & Cáncer gástrico & $3 \%$ & \\
\hline & Cáncer cérvix & $1 \%$ & \\
\hline & Cáncer colon & $1 \%$ & \\
\hline & Cáncer ovario & $1 \%$ & \\
\hline & Cáncer pulmón & $1 \%$ & \\
\hline & Cáncer testículo & $1 \%$ & \\
\hline & Osteosarcoma & $1 \%$ & \\
\hline
\end{tabular}

La mayoría de los pacientes (79\% de los casos) no presentaba una comorbilidad asociada a la neoplasia maligna; el 12 \% de los casos que presentó neutropenia febril tenían una patología asociada, siendo las más frecuentes HTA, diabetes mellitus, falla cardiaca y EPOC.

Las terapias sistémicas más frecuentemente relacionadas a casos de neutropenia febril, fueron los esquemas de quimioterapia combinada empleados para el tratamiento de las leucemias agudas y linfomas no Hodgkin, que constituyen el $40 \%$ y el $22 \%$ de los casos respectivamente. Entre las terapias utilizadas para el tratamiento de neoplasias sólidas más asociadas a neutropenia febril, se encontraron el esquema de quimioterapia AC ( $4 \%$ de los casos) y el régimen carboplatino más paclitaxel (3\%). El $25 \%$ de los casos de neutropenia febril recibió profilaxis primaria con factores estimulantes de granulocitos (Tabla 3).

Se realizó hemocultivo al 93.4 \% de los pacientes con neutropenia febril, de los cuales en el $56 \%$ no se detectaron gérmenes y en el $44 \%$ de los casos los resultados fueron positivos. Los bacilos Gram negativos fueron predominantes ( $59 \%$ de los casos), Escherichia coli en el $33 \%$ de
Tabla 3. Terapias sistémicas (quimioterapia y terapias blanco) relacionadas con los episodios de neutropenia febril.

\begin{tabular}{|l|l|}
\hline Terapias & Total \\
\hline BFM & $17 \%$ \\
\hline RITUXIMAB+CHOP & $16 \%$ \\
\hline CITARABINA & $14 \%$ \\
\hline IDA - FLAG & $9 \%$ \\
\hline HYPER-CVAD & $7 \%$ \\
\hline AC & $4 \%$ \\
\hline AZACITIDINA & $3 \%$ \\
\hline GMALL & $3 \%$ \\
\hline RITUXIMAB+CVP & $3 \%$ \\
\hline CARBOPLATINO +PACLITAXEL & $3 \%$ \\
\hline DCF & $3 \%$ \\
\hline CICLOFOSFAMIDA + RITUXIMAB & $1 \%$ \\
\hline CYBORD & $1 \%$ \\
\hline METOTREXATE+VINCRISTINA & $1 \%$ \\
\hline ASPARAGINASA & $1 \%$ \\
\hline RITUXIMAB +FLUDARABINA & $1 \%$ \\
\hline TRASTUZUMAB EMTANSINE & $1 \%$ \\
\hline DHAP & $1 \%$ \\
\hline $\begin{array}{l}\text { CITARABINA } \\
\text { +DAUNORRUBICINA }\end{array}$ & $1 \%$ \\
\hline DOXORRINCINA + VINCRISTINA & $1 \%$ \\
\hline CLADRIBINE & $1 \%$ \\
\hline EMA-CO & $1 \%$ \\
\hline TIP & $1 \%$ \\
\hline FOLFOX 6 & $1 \%$ \\
\hline CISPLATINO +DOXORRUBICINA & $1 \%$ \\
\hline ABVD & $1 \%$ \\
\hline CISPLATINO + ETOPOSIDO & $1 \%$ \\
\hline
\end{tabular}

Ios casos y Pseudomonas aeruginosa y KlebsieIla pneumoniae, seguido por los cocos Gram positivos ( $27 \%$ de los casos), principalmente Staphylococcus aureus. Se documentaron especies de Candida en hemocultivos, en el $3 \%$ de casos (Figura 2).

La mayoría de los pacientes (82 \%) que desarrollaron neutropenia febril no tuvieron necesidad de ingresar a UCl y solo el $18 \%$ de los casos (14 pacientes) con neutropenia febril requirieron hospitalización en $\mathrm{UCl}$. De los pacientes que fueron trasladados a UCl, el 92 \% (13 casos) presentaban una neoplasia hematológica maligna. La tasa de mortalidad de los pacientes con neutropenia febril que requirieron hospitalización 


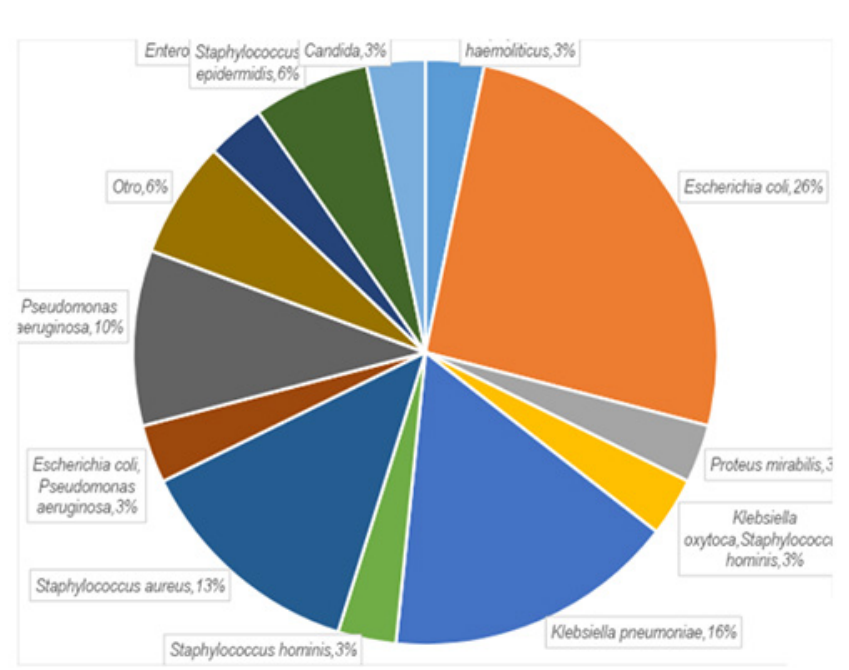

Figura 2. Perfil microbiológico en los sujetos con hemocultivos positivos.

en UCl fue del $57 \%$ (8 casos).

El promedio de resolución de la neutropenia febril fue de 9.2 días, con una desviación estándar de 7.7 días; la estancia promedio hospitalaria de los pacientes que presentaron neutropenia febril fue de 20.7 días y la duración promedio de la antibioticoterapia fue de 11.8 días.

La tasa de mortalidad en los pacientes que presentaron neutropenia febril fue del $14 \%$ (11 pacientes en total). De los pacientes que fallecieron, el $81 \%$ de los casos (9) tenían como enfermedad de base neoplasia hematológica.

En el presente estudio la tasa de mortalidad asociada a neutropenia febril fue del $11.8 \%$ frente a un $2.6 \%$ entre los que tenían diagnóstico de neoplasias hematológicas y neoplasias sólidas malignas, respectivamente.

\section{Discusión}

A pesar de los avances en efectividad y seguridad de las terapias oncológicas, la neutropenia febril permanece como una de las complicaciones más frecuentes y severas de la quimioterapia, convirtiéndose en una disminución de la efectividad de la quimioterapia por retrasos en las dosis de los fármacos y, aunque ha disminuido su mortalidad, permanece siendo significativa ${ }^{3}$.

Este estudio es el primero en describir en población adulta con neoplasias hematooncólogicas, las características clínico-patológicas y los hallazgos microbiológicos de la neutropenia febril en pacientes de la costa caribe colombiana.

La edad promedio de los casos con neutropenia febril fue 43 años, cifra que está en el rango de edad promedio reportado en los estudios realizados en la población colombiana (41 a 50 años) ${ }^{10,11,12,13,14}$. La presencia de neutropenia febril fue similar en ambos sexos, con una relación hombre: mujer de 1.2:1.

La neutropenia febril se presentó con mayor frecuencia en pacientes con neoplasias hematológicas (84.2\%), lo cual está asociado a que esta población recibe esquemas de quimioterapia que ocasionan mayores tasas de mielo supresión severa.

La tasa de documentación clínica o imagenológica de infección en pacientes con neutropenia febril es variable de un $30 \%$ a un $60 \%{ }^{15,16}$, en el presente estudio se evidenció foco infeccioso de la neutropenia febril en el 68 \% de los casos, siendo los más frecuentemente documentados: tracto respiratorio ( $21 \%$ de los casos), tracto digestivo (12\%) y piel/tejidos blandos (12\% de los casos), resultados similares a lo reportado en dos estudios nacionales realizados recientemente. El primero en un grupo de centros oncológicos del eje cafetero, el cual reportó un 69 $\%$ de casos con foco infeccioso ${ }^{12}$ y el segundo realizado en el Hospital San Ignacio de Bogotá, el cual documentó foco infeccioso en el $68.1 \%$ de los episodios ${ }^{13}$.

Respecto a los hallazgos microbiológicos se encontró que entre los pacientes a los cuales se les realizó hemocultivos (93.4\% de los casos), la positividad fue del $44 \%$, siendo los gérmenes más frecuentes los bacilos Gram negativos (59\% de los casos), y entre ellos los de mayor incidencia fueron Escherichia coli, Pseudomonas aeruginosa y Klebsiella pneumoniae; seguido por los cocos Gram positivos (27\% de los casos) Staphylocococcus aureus.

Con relación al perfil microbiológico de la neutropenia febril, este ha variado en el tiempo; inicialmente la mayoría de los gérmenes aislados eran bacilos Gram negativos, posteriormente a finales del siglo pasado y principios del actual, se presentó un cambio a gérmenes Gram positivos y actualmente en las dos últimas déca- 
das, nuevamente existe predominio de bacilos Gram negativos.

Los resultados del presente estudio están en consonancia con estos hallazgos y con lo reportado en varios estudios nacionales, los cuales evidenciaron que los gérmenes Gram negativos son la etiología microbiológica más frecuente de neutropenia febril, con una tasa de aislamiento entre $49 \%$ y $75 \% 10,11,12,13,14$.

Se evidenció una baja tasa de profilaxis primaria con factores estimulantes de colonias de granulocitos; sólo el $25 \%$ de los casos de neutropenia febril la recibieron, teniendo en cuenta que la mayoría de los pacientes que presentaron este evento secundario recibieron quimioterapias de alta intensidad, empleadas para el tratamiento de las leucemias agudas y linfomas no Hodgkin que constituyeron el $62 \%$ de los casos.

Un estudio retrospectivo en pacientes con cáncer de seno, pulmón, ovario, colon/recto y Linfoma no Hodgkin con riesgo intermedio/alto para neutropenia febril, recibieron profilaxis primaria con factores estimulantes de colonias de granulocitos (pegfilgrastim $>96 \%$ casos) en el $52 \%$ de los casos en el año 2010 y con ligero aumento del uso a un $58 \%$ de los eventos en el año $2016{ }^{17}$.

La tasa de mortalidad de la neutropenia febril obtenida en este estudio fue de $14 \%$, siendo del $11.8 \%$ en pacientes con neoplasias hematológicas y del $2.6 \%$ entre aquellos con neoplasias sólidas malignas. Estos resultados están en el rango de lo reportado en estudios realizados en instituciones colombianas (7 \% a 16.5 \%) 10,11,12,13,14, y las tasas de mortalidad son similares a las descritas a nivel mundial 10-12.5\% 3,16 .

La principal limitación del presente estudio fue la falta de disponibilidad de información más completa en la historia clínica, dada la naturaleza retrospectiva de los datos obtenidos, especialmente la ausencia de la clasificación MASCC de neutropenia febril en un alto porcentaje de los registros, lo cual no permitió reportar este importante factor pronóstico.
El presente estudio contribuye a la obtención de conocimiento sobre las características clínico-patológicas y los hallazgos microbiológicos de la neutropenia febril, en pacientes con neoplasias hematooncológicas atendidos en el Instituto Médico de Alta Tecnología (IMAT) en la ciudad de Montería-Colombia, el cual es centro de referencia para estas patologías en los departamentos de Córdoba y Sucre.

Se encontraron características clínicas y microbiológicas similares a lo reportado en instituciones en Colombia y resultados clínicos, como tasa de mortalidad, semejantes a lo descrito a nivel nacional e internacional.

\section{Conclusiones}

El presente estudio realizado en pacientes con neoplasias hematooncólogicas de la costa caribe colombiana, evidenció una alta tasa de documentación de foco infeccioso de la neutropenia febril. Se obtuvo desarrollo bacteriano en los hemocultivos en el $44 \%$ de los casos, siendo los bacilos Gram negativos, seguido por los cocos Gram positivos en el $27 \%$ de los casos los microorganismos responsables. La tasa de mortalidad obtenida fue del $14 \%$, resultados similares a los reportados por instituciones colombianas (7 \% a $16.5 \%$ ) y a lo descrito en población latinoamericana y a nivel mundial (10-12.5\%).

- Aspectos Éticos: El estudio fue conducido de acuerdo con la reglamentación vigente sobre ética en investigación y fue aprobado por el comité de ética institucional. Dado que la información se obtuvo de forma retrospectiva, no fue necesaria la implementación del consentimiento informado.

- Fuente de Financiación: El estudio fue realizado con recursos aportados por AMGEN.

- Conflictos de Interés: Los autores manifiestan presentar el conflicto de interés derivado del patrocinio económico recibido para la realización del estudio. 


\section{Referencias}

1. Gayol M, Font A, Casas I, Estrada O, Domínguez $M$, Botet P. Utilidad de la escala de MASCC en el tratamiento de neutropenia febril inducida por quimioterapia en pacientes con neoplasia sólida. Med Clin (Barc) [Internet]. 2009; 133 (8): 296-299. https://doi.org/10.1016/j.medcli.2008.12.022

2. Freifeld A, Bow E, Sepkowitz K, Boeckh M, Ito J, Mullen C, et al. Clinical Practice Guideline for the Use of Antimicrobial Agent in Neutropenic patients with Cancer: 2010 Update by the Infectious Diseases Society of America. Clinical Infectious Diseases [Internet]. 2011; 52 (4):56-93. https://doi. org/10.1093/cid/cir073

3. Klastersky J, Naurois J, Rolston K, Rapoport B, Maschmeyer G, Aapro M, et al. Management of febrile neutropenia: ESMO Clinical Practice Guidelines. Annals of Oncology [Internet]. 2016; 27(Suppl 5): v111-v118. https://doi.org/10.1093/annonc/ mdw325

4. González X, Gamba J, Bolaños J, Villeta L. Aislamientos microbiológicos en pacientes con neutropenia febril. ¿Es apropiado el uso de las guías clínicas internacionales en México? Rev Hematol Mex. [Internet]. 2013; 14: 113-119. https://bit.ly/30fXyFf

5. Rasmy A, Amal A, Fotih S, Selwi W. Febrile neutropenia in cancer patient: Epidemiology, microbiology, pathophysiology and management. Journal of Cancer Prevention \& Current Research [Internet]. 2016;5(3):00165. https://doi.org/10.15406/ jcpcr.2016.05.00165

6. Klastersky J. Raftopoulos H, Rapoport B. The MASCC Neutropenia, Infection and Myelosuppression Study Group evaluates recent new concepts for the use of granulocyte colony-stimulating factors for the prevention of febrile neutropenia. Support Care Cáncer [Internet]. 2013; 21: 1793-1795. https://doi.org/10.1007/s00520-0131776-9

7. Maldonado S, Bermúdez L, Gómez J, Castillo J, Sánchez R, Ballesteros $M$, et al. Guía de práctica clínica para el diagnóstico y el tratamiento de las infecciones bacterianas y micóticas en pacientes oncológicos mayores de 15 años con neutropenia febril posquimioterapia de alto riesgo. Rev Colomb Cancerol [Internet]. 2014; 18 (4): 186-196. http://dx.doi.org/10.1016/j.rccan.2014.06.002

8. White L, Ybarra M. Neutropenic fever. Emerg Med Clin N Am 2014; 32: 549-561. https://doi.org/10.1016/j.emc.2014.04.002

9. Aapro MS, Bohlius J, Cameron DA, Dal Lago L,
Donnelly JP, Kearney N, et al. 2010 update of ECRTC guidelines for the use granulocytes-colony stimulating factor to reduce the incidence of chemotherapy-induced febrile neutropenia in adult patients with lymphoproliferative disorders and solid tumours. Eur J Cancer [Internet]. 2011; 47: 8-32. https://doi.org/10.1016/j.ejca.2010.10.013

10. García K, Londoño J, Villegas L, González M, Correa A. Diagnóstico microbiológico en neutropenia febril secundaria a quimioterapia por malignidad hematológica. Acta Médica Colombiana [Internet]. 2020; 45:1-7. https://doi.org/10.36104/ amc.2020.1386

11. Arroyave T, Puerta J, Beltran R, Salgado T, Ramirez $\mathrm{D}$, Arroyave $\mathrm{D}$, et al. Características de los pacientes adultos con neutropenia febril en un hospital universitario (Medellín, 2012-2016). Medicina UPB [Internet]. 2019; 38(2): 108-113. https://doi. org/10.18566/medupb.v38n2.a02

12. Cataño D, Marín D, Rivera J, Martínez J, Sánchez J, et al. Neutropenia febril asociada a quimioterapia en pacientes con neoplasias hematológicas de un centro de referencia en Colombia: Características clínicas y desenlaces. SaludUninorte [Internet]. 2020 35(2):205-220. http://dx.doi. org/10.14482/sun.35.2.616.15

13. Garzón J, Isaza N, Posada A, Méndez R, Rodríguez M, Ardila MP, et al. Características clínicas y microbiológicas de pacientes con neutropenia febril en un hospital universitario. Infectio [Internet]. 2019; 23(4): 347-357. http://dx.doi.org/10.22354/ in.v23i4.806

14. Ramos P, Sánchez R, Gamboa O, Cardona A. Factores pronósticos relacionados con la mortalidad en pacientes con cáncer y neutropenia febril. Revista Colombiana de Hematología y Oncología. 2012; 1(4):15-22

15. Schelenz S, Giles D, Abdallah S. Epidemiology, management and economic impact of febrile neutropenia in oncology patients receiving routine care at a regional UK cancer centre. Ann of Oncology [Internet]. 2012; 23: 1889-1893. https:// doi.org/10.1093/annonc/mdr520

16. Punnapuzha S, Edemobi PK, Elmoheen A. 2019 Dec 30. In: StatPearls [Internet]. Treasure Island (FL): StatPearls Publishing; 2020 Jan

17. Weycker D, Bensink M, Lonshteyn A, Doroff R, Chandler D. Use of colony stimulating factor primary prophilaxis and incidence of febrile neutropenia from 2010-2016: a longitudinal assesment. Curr Med Res Opin [Internet]. 2019; 35(6): 10731080. https://doi.org/10.1080/03007995.2018.1558 851 